\title{
Crystallography of Intermetallic Interface Layers in Hot-dip Galvanizing Steel Sheets
}

\author{
Hiroyuki OHTSUBO, Takahide YAGI," Kiyomichi NAKAl and Yasuya OHMORI
}

Department of Materials Science \& Engineering, Ehime University, Bunkyo-cho, Matsuyama, Ehime-ken, 790 Japan. 1) Formerly Graduate Student, Ehime University. Now at Sumiju Examination and Inspection Co., Ltd., Toyo, Ehime-ken, Japan.

(Received on August 17, 1995; accepted in final form on September 25, 1995)

\begin{abstract}
The crystallography of the intermetallic phases forming at the zinc-film/substrate interfaces in hot-dip galvanizing steel sheets has been investigated mainly by means of scanning electron and cross sectional transmission electron microscopies. Zinc atoms penetrating into a ferrite lattice precipitate within the ferrite as monoclinic $\zeta$-phase rods with parallelogram cross sections initially in contact with the interface. The growth direction of the $\zeta$-phase rod is probably the $[001]_{\zeta}$ which lies parallel to a $\langle 111\rangle_{\alpha}$. The orientation relationship between $\zeta$ and the substrate ferrite, $[001]_{\zeta} / /[111]_{\alpha^{\prime}}(100)_{\zeta} / /(101)_{\alpha^{\prime}}(010)_{\zeta} / /(12 \overline{1})_{\alpha^{\prime}}$ can be expected by considering both the results of scanning microscopy and the atomic configurations of $\zeta$ phase and those of ferrite. The additional heat cycle up to $723 \mathrm{~K}$ to produce thin foils for cross sectional transmission electron microscopy after the hot-dip galvanizing process induces the formation of $\delta_{1}$-phase at the ferrite/ $\zeta$ interphase boundary. No specific orientation relationship between the $\delta_{1}$-phase and the substrate ferrite was obtained.
\end{abstract}

KEY WORDS: hot-dip galvanizing; surface treatment; Zn-plating; intermetallic layers; crystallography of intermetallic layers; epitaxy of intermetallic layers.

\section{Introduction}

It has been well established that an electrodeposited metallic film grows epitaxially on a metallic substrate. ${ }^{1-10)}$ In the case of hot-dip galvanizing, however, since many intermetallic layers such as $\mathrm{FeZn}_{13}(\zeta)$, $\mathrm{FeZn}_{7}\left(\delta_{1}\right), \mathrm{FeZn}_{4}\left(\Gamma_{1}\right)$ and $\mathrm{Fe}_{3} \mathrm{Zn}_{10}(\Gamma)$ are formed between $\eta$-zinc film and a steel substrate, ${ }^{11)}$ the mutual orientation relationships are difficult to examine, the epitaxy being vaguely understood.

According to the experiment by Scheil, ${ }^{12)}$ the $\mathrm{Fe}-\mathrm{Zn}$ intermetallic crystals nucleating at the interface between molten $\mathrm{Zn}$ and ferrite grow into the molten $\mathrm{Zn}$ macroscopically with very flat advancing surfaces almost parallel to that of the substrate in the direction normal to the ferrite surface. If the intermetallic phases precipitated at a ferrite interface within the molten $\mathrm{Zn}$ with the consuming of Fe atoms dissolving in the molten $\mathrm{Zn}$, the intermetallic crystals in dendritic morphology would be resulted. Thus such a flat morphology of the advancing interfaces would favour the interpretation that the ferrite lattices where $\mathrm{Zn}$ atoms penetrated transform into the intermetallic lattices, the volume expansion due to this reaction leading to the morphology as if they grow into the molten $\mathrm{Zn}$. In such a case where the formation of the intermetallic phase is a solid phase reaction, the intermetallic crystals will grow epitaxially within the substrate as far as the atomic configurations in the intermetallic phase are similar to those in the substrate. In fact, some evidences showing the epitaxial growth of $\zeta$-phase crystals has been reported by Adachi et al. ${ }^{13-15)}$ in the hot-dip galvanizing steels with the addition of small amounts of $\mathrm{Al}$. It has also been pointed out ${ }^{16)}$ that $\mathrm{Al}$ atoms interact with $\mathrm{Fe}$ atoms at the substrate surface and form a barrier layer preventing the direct reaction between the molten $\mathrm{Zn}$ atoms and $\mathrm{Fe}$ atoms on the substrate ferrite surface. Thus, $\mathrm{Zn}$ atoms penetrating into the Fe lattice through this barrier layer is thought to react with $\mathrm{Fe}$ atoms and precipitate intermetallic phases. The crystallographic analyses carried out so far, however, still involve some ambiguous points because of the difficulties in preparing thin foils for transmission electron microscopy.

Intermetallic layers described above can also be formed in the $\mathrm{Zn}$-electrodeposited steel sheets annealed at various temperatures. In such cases, Gannuzzi et $a l .^{17-20)}$ obtained the lattice image for the (0001) planes of $\delta_{1}$ phase. Lin et al. ${ }^{21)}$ also observed the structural images of $\Gamma / \alpha-\mathrm{Fe}$ and $\Gamma / \delta_{1}$ interfaces and determined the crystallographic relationships between them. It is, however, still questionable that these relationships can also be applicable to the hot dip galvanizing case.

The aims of the present study, therefore, are to reexamine the crystallographic features between the intermetallic phases and the substrate, and to reveal the mechanisms of their formation in hot-dip galvanizing 
steel sheets.

\section{Experimental Procedures}

Material used as the substrate is a $1 \mathrm{~mm}$ thick low carbon steel sheet. The specimens were prepared by machining it into the shape $8 \mathrm{~mm}$ wide and $140 \mathrm{~mm}$ long. These specimens were encapsulated into vacuum quartz tubes, annealed at $1073 \mathrm{~K}$ for $10.8 \mathrm{ks}$ and were supplied as the polycrystal substrates. Some of the annealed specimens were strained $1 \%$ by a tensile machine and were again annealed at $1153 \mathrm{~K}$ for $259.2 \mathrm{ks}$ after encapsulating into vacuum quartz tubes to get extremely large crystals. The orientation of these large crystals were determined by X-ray back reflection Laue technique. The target used was molybdenum, and the acceleration voltage, the current and the exposing time were $30 \mathrm{kV}, 18 \mathrm{~mA}$ and $10.8 \mathrm{ks}$, respectively. The substrates prepared as above were polished chemically in $\mathrm{H}_{2} \mathrm{O}_{2}$ water saturated by $(\mathrm{COOH})_{2}$, and then they were immersed into the $50 \% \mathrm{ZnCl}_{2}$ flux solution. After drying at $373 \mathrm{~K}$, they were dipped into $\mathrm{Zn}$-bath containing $0.1 \% \mathrm{Al}$ kept at $733 \mathrm{~K}$ for $2 \mathrm{~s}$. This treatment produced the surface film comprising $\eta$-phase and an intermetallic layer.

In order to examine the morphology of the intermetallic layer, the outermost $\eta$-phase layer was dissolved by chemical polishing in dilute $\mathrm{HCl}$ solution ${ }^{13)}$ and the morphology of intermetallic crystals was observed by a scanning electron microscope. The crystal structure was determined by X-ray diffractometer $(\mathrm{Cu}-\mathrm{K} \alpha, 35 \mathrm{kV}$, $15 \mathrm{~mA}$ ).

Thin foils for cross sectional transmission electron microscopy were prepared as follows. Five hot-dip galvanized specimens were mutually stacked and were again dipped into hot $\mathrm{Zn}$-bath kept at $723 \mathrm{~K}$ for $2 \mathrm{~s}$ to bond to each other by remelting the $\mathrm{Zn}$ surfaces. These stacked specimens were sliced vertically by a microslicing machine and were polished mechanically by a dimpling machine. The final polishing was achieved by means of ion milling technique. Thin foils were observed by JEM-2000EX microscope operated at $200 \mathrm{kV}$.

\section{Experimental Results}

Figure 1 shows the cross sectional view observed by a scanning electron microscope for the hot-dip galvanized specimen lightly etched. The cross section can be divided clearly into three regions. The outermost surface and the bottom substrate are of course the $\eta$-zinc layer and the substrate ferrite, respectively. Many etch pits can be observed in the $\eta$-zinc layer. The intermediate is the intermetallic layer. It should be noted that, although the interface between the intermetallic layer and the substrate is quite flat, the boundary separating the $\eta$-zinc film and the intermetallic layer exhibits sawteethlike morphology with specific orientations as has been pointed out by Onishi et $a .^{22)}$ This implies that the orientation of the intermetallic layer is relevant to the substrate orientation, the epitaxy between the intermetallic layer and the substrate existing. The result of $\mathrm{X}$-ray diffraction from the specimen surface of which

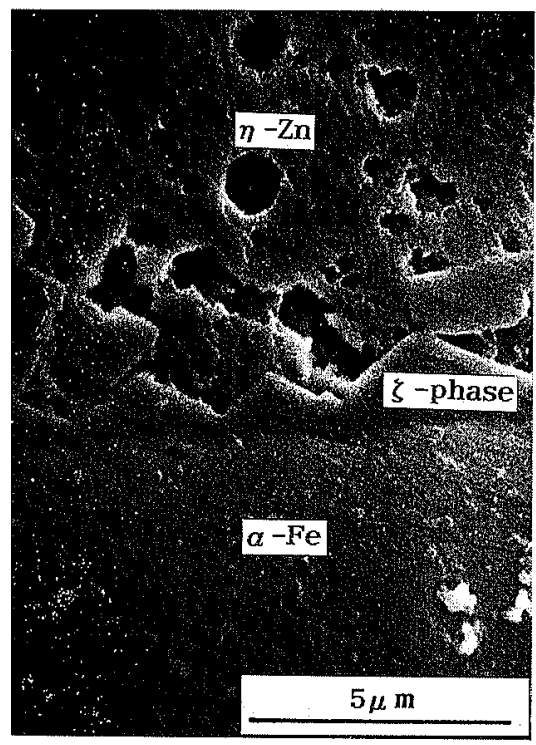

Fig. 1. The cross section of an as-hot-dipped galvanizing steel sheet. The specimen was dipped for $2 \mathrm{~s}$ in the $\mathrm{Zn}$-bath kept at $733 \mathrm{~K}$.

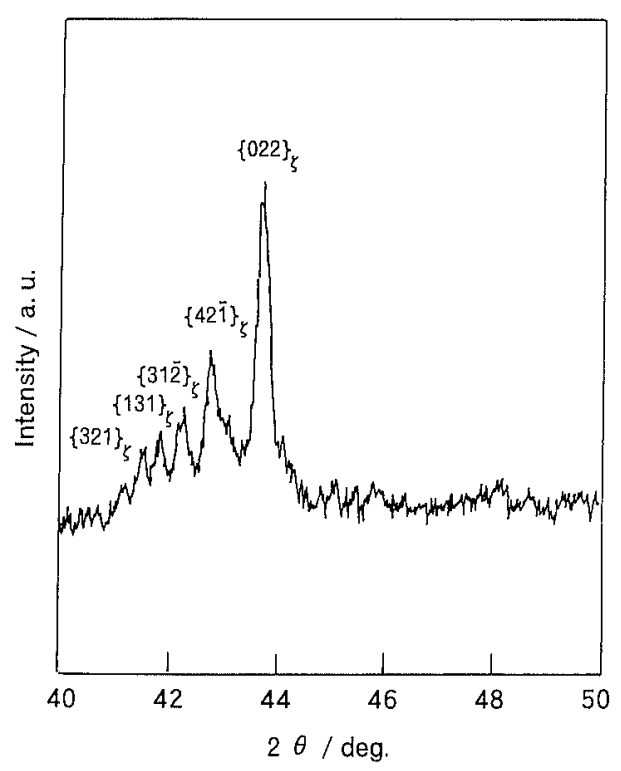

Fig. 2. The X-ray diffraction profile from the surface of the as-hot-dipped specimen. $\eta$-zine layer was dissolved by light etching in dilute $\mathrm{HCl}$ solution. Diffracted lines can be identified as those from $\zeta$-phase.

$\eta$-zinc layer was dissolved in dilute $\mathrm{HCl}$ solution is shown in Fig. 2. All the reflections can be identified as FeZn $\mathrm{Zn}_{13}$ $\zeta$-phase. This is in keeping with the scanning electron micrograph shown in Fig. 1 where only one intermetallic layer was recognized. Thus the initially formed intermetallic phase at $733 \mathrm{~K}$ except very thin Al-enriched layer which could not be identified in the present study is thought to be $\zeta$-phase. Occasionally, the so-called out-burst reaction ${ }^{23,24)}$ where the $\zeta$-phase layer is broken into fragments within the outer $\eta$-zinc layer was also observed as shown in Fig. 3.

Figure 4 shows the microstructural correspondence between the ferrite grains of the substrate and the morphology of $\zeta$-phase crystals revealed by light etching. Grain boundaries of the polycrystal ferrite substrate 


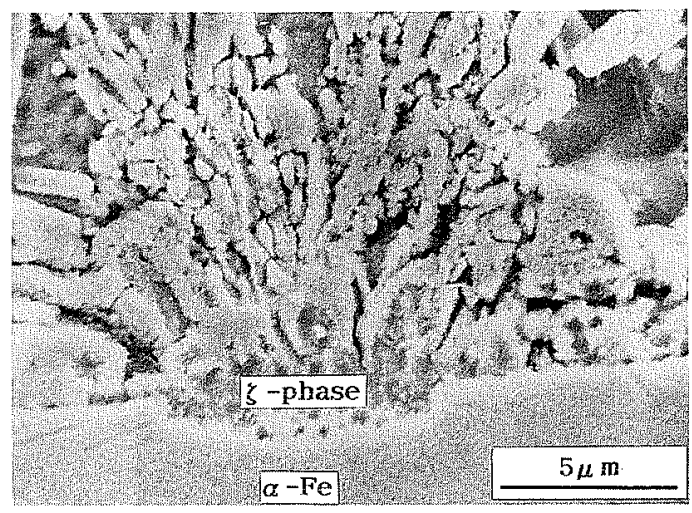

Fig. 3. Out-burst reaction observed in the specimen hotdipped in a $\mathrm{Zn}$-bath kept at $733 \mathrm{~K}$ for $2 \mathrm{~s}$.

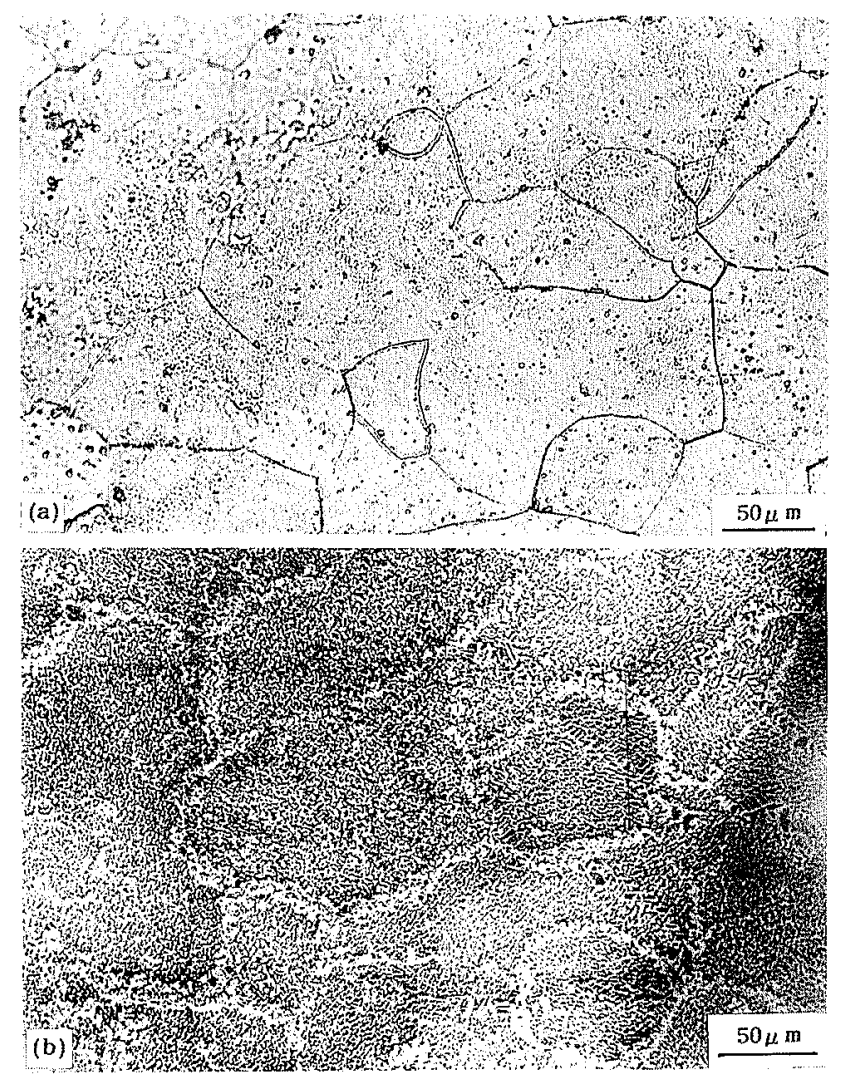

Fig. 4. Microstructural correspondence between the substrate ferrite and the $\zeta$-phase crystals formed by the immersion into the $\mathrm{Zn}$-bath kept at $733 \mathrm{~K}$ for $2 \mathrm{~s}$.

(a) ferrite grain boundaries revealed by etching of the Fe substrate and (b) $\zeta$-phase crystals formed on it.

can clearly be seen in Fig. 4(a). This specimen was dipped in the hot $\mathrm{Zn}$-bath held at $723 \mathrm{~K}$ for $2 \mathrm{~s}$. After dissolving the outermost $\eta$-zinc layer in dilute $\mathrm{HCl}$ solution, the morphology of $\zeta$-crystals was observed. The result is shown in Fig. 4(b). ऊ-phase crystals are aligned in specific orientations within each ferrite grain, showing the epitaxy between the $\zeta$-phase crystals and the substrate ferrite grains. At ferrite grain boundaries, $\zeta$-phase crystals were randomly orientated. It should also be noted that the ferrite grain boundaries on the initial substrate surface shown in (a) do not precisely coincide with those in (b). This implies that the grain boundaries observed in (b) were located at the layer somewhat below the original substrate surface, i.e., the

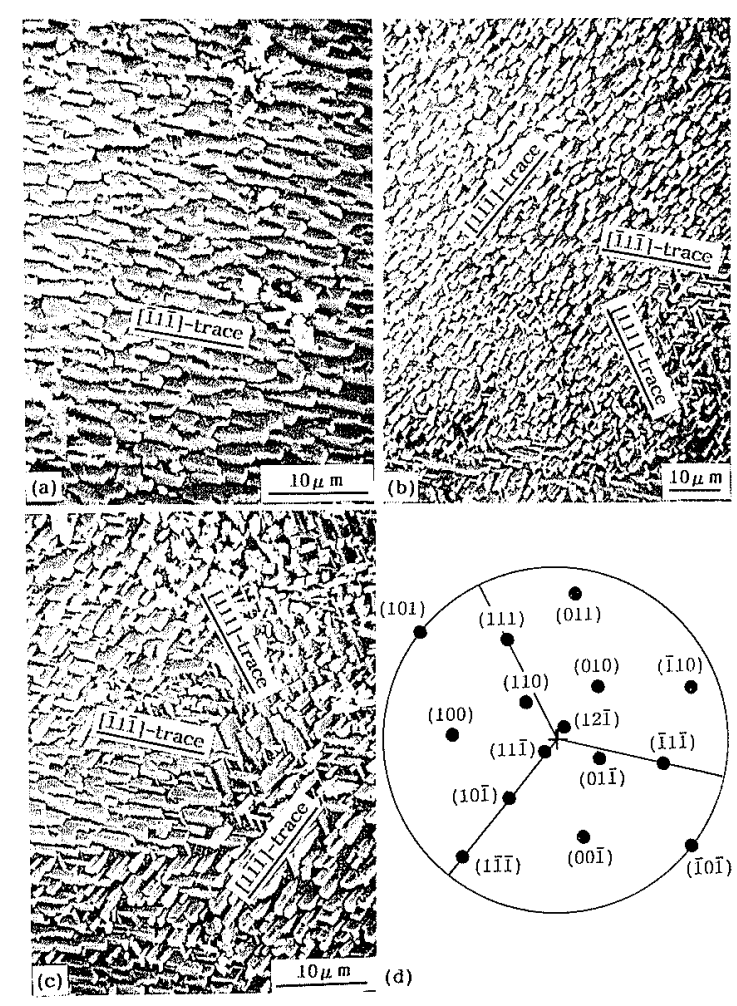

Fig. 5. Morphology of $\zeta$-phase crystals on various locations within a ferrite grain.

(a) single growth direction, (b) three different growth directions, (c) four growth directions, and (d) the stereogram showing the orientation of the ferrite substrate.

$\zeta$-phase crystals did not precipitate directly on the ferrite surface but formed within the ferrite grains slightly below from the surface by the penetration of $\mathrm{Zn}$ atoms into the ferrite by diffusion as has been postulated previously. ${ }^{16,25)}$ In order to reveal the detailed configuration of $\zeta$-phase crystals, similar observation was carried out on a very coarse ferrite grain of which orientation was preliminarily determined by X-ray back Laue reflections. The results are shown in Fig. 5. Figures $5(\mathrm{a})$ to 5 (c) illustrate the morphology of the $\zeta$-phase crystals formed in the same ferrite grain. The orientation of the ferrite substrate is shown in the stereogram (d). The crystallographic plane parallel to the substrate surface is in the midway between the $(12 \overline{1})_{\alpha}$ and the $(11 \overline{1})_{\alpha}$. The number of the variants of $\zeta$-phase growth directions within this grain are different locally, i.e., single variant in (a), three variants in (b) and four variants in (c). It is interesting to note that the growth directions of $\zeta$-phase crystals observed in a ferrite grain of various orientation are four at the maximum and the traces of them always pass through the $\langle 111\rangle_{\alpha}$ poles in the ferrite stereogram, suggesting that the growth direction is parallel to $\langle 111\rangle_{\alpha}$ of ferrite. Trace normal analysis for the growth directions of $\zeta$-phase crystal in a ferrite $(001)_{\alpha}$ stereogram is illustrated in Fig. 6. The large circles $90^{\circ}$ from the trace normal poles focus at the $[111]_{\alpha}$. Therefore, the growth direction of a $\zeta$-phase crystal is parallel to a $\langle 111\rangle_{\alpha}$ direction. This growth direction is also consistent with the result that the maximum variants of growth directions in a specific 


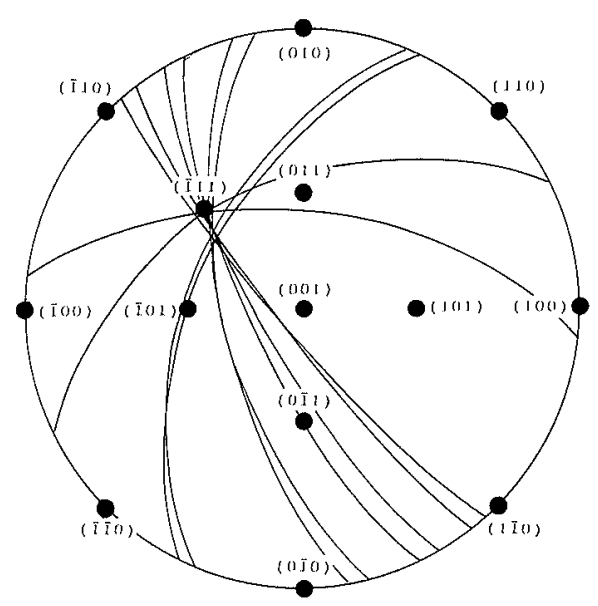

Fig. 6. Trace normal analysis for the growth direction of $\zeta$-phase crystal.

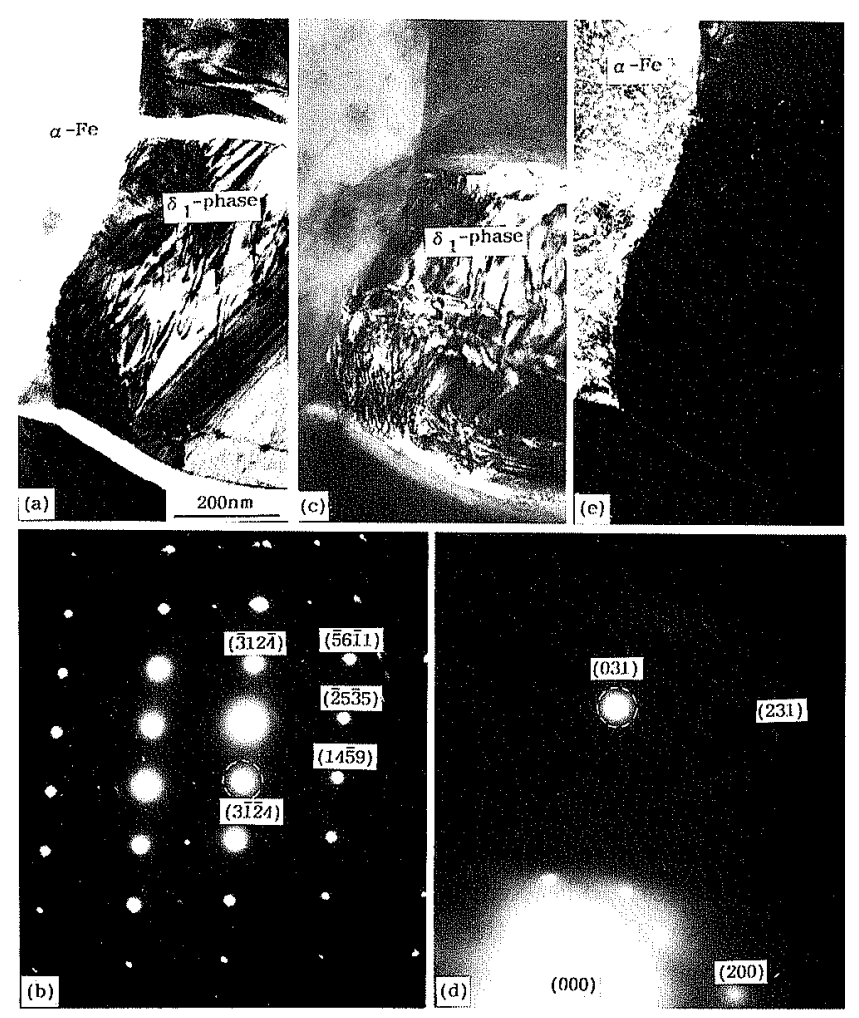

Fig. 7. Transmission electron micrographs showing the $\delta_{1}$-phase/ferrite interface. The reffections used for dark field illuminations were encircled by broken lines. (a) the bright field image, (b) the selected area electron diffraction pattern from a $\delta_{1}$-phase crystal, (c) the dark field image of the $\delta_{1}$-phase crystal using the $(3 \overline{1} 4)_{\delta}$, (d) the selected area electron diffraction pattern from the ferrite in contact with the $\delta_{1}$-phase crystal, and (d) the dark field image of the ferrite using the $(031)_{\alpha}$.

ferrite grain are four, i.e., $[111]_{\alpha},[\overline{1} 11]_{\alpha},[1 \overline{1} 1]_{\alpha}$ and $[11 \overline{1}]_{\alpha}$. If the growth direction of $\zeta$-phase crystal were of $\langle h h l\rangle_{\alpha}$ type ( $h$ and $l$ are integers, and $h \neq l$ ) as has been reported, ${ }^{13)}$ the maximum equivalent growth directions in a ferrite would be twelve and more than four variants of growth directions could be observed.

The cross sectional transmission electron microscopy was also carried out to reveal the interface structures between the intermetallic layers and the substrate. The intermetallic layers observed in contact with the ferrite,
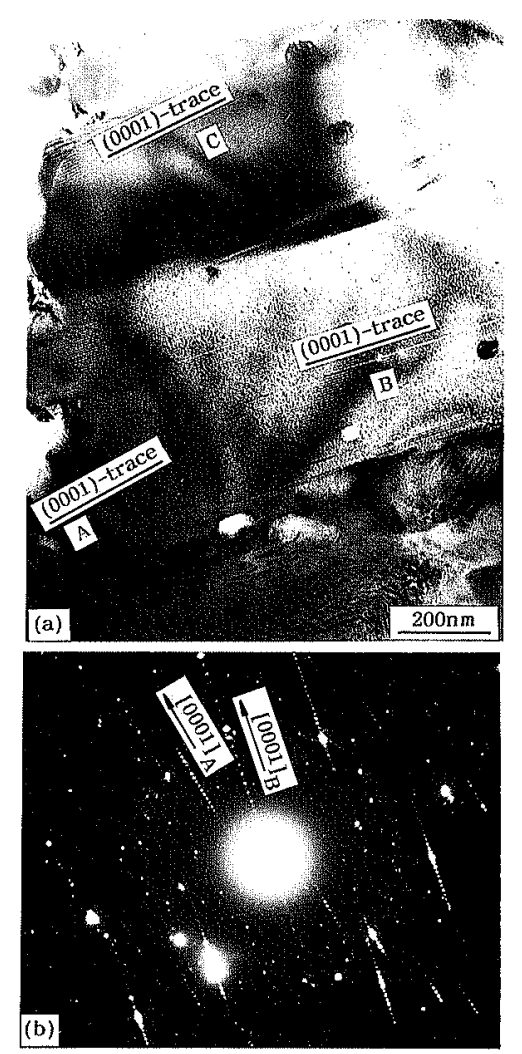

Fig. 8. Transmission electron micrograph showing the $\delta_{1^{-}}$ phase/ferrite interface structure. The regions indicated as $\mathrm{A}, \mathrm{B}$ and $\mathrm{C}$ provide different $(0001)$ lattice images. (a) the $(0001)_{\delta}$ lattice images, (b) the selected area electron diffraction pattern.

however, were confirmed to be $\delta_{1}$-phase unfortunately. This situation is thought to occur probably due to the additional heat cycle during the preparation of thin foils. Figure 7 shows the interface between a $\delta_{1}$-phase crystal and the Fe substrate. Figures 7(a), 7(b) and 7(c) are the bright field image, the selected area electron diffraction pattern from the $\delta_{1}$-phase crystal and the dark field image using the $(3 \overline{1} \overline{2} 4)_{\delta}$ reflection, respectively. The selected area diffraction from the ferrite grain in contact with the $\delta_{1}$-phase crystal and the dark field image using the $(031)_{\alpha}$ are in Figs. 7(d) and 7(e), respectively. Many diffraction patterns from the $\delta_{1}$ crystals in contact with $\alpha$-ferrite grains were also examined, but a specific orientation relationship cannot be determined. An example of this situation is shown below. Since the $c$-axis of $\delta_{1}$ lattice is extremely large, i.e., $5.735 \mathrm{~nm}$, the lattice images of them can frequently be observed even at relatively low magnification. Figure 8 shows the $\delta_{1}$ phase/ferrite interface structure. Since the interplanar spacing of $(0001)_{\delta}$ planes is very large, some of $(000 k)_{\delta}$ reflections ( $k$ 's are integers) are involved within the objective aperture when the $[0001]_{\delta}$ directions are normal to the incident electron beam, leading to the formation of $(000 k)_{\delta}$ lattice images. Figure 8(a) shows the bright field image at the interface between $\delta_{1}$-phase crystals and the substrate ferrite. Two sets of the $(0001)_{\delta}$ lattice images can clearly be seen. The selected area electron diffraction pattern is in Fig. 8(b). In this pattern, also two sets of the $(000 k)_{\delta}$ reflections aligning in slightly different orientations in accordance with the lattice 


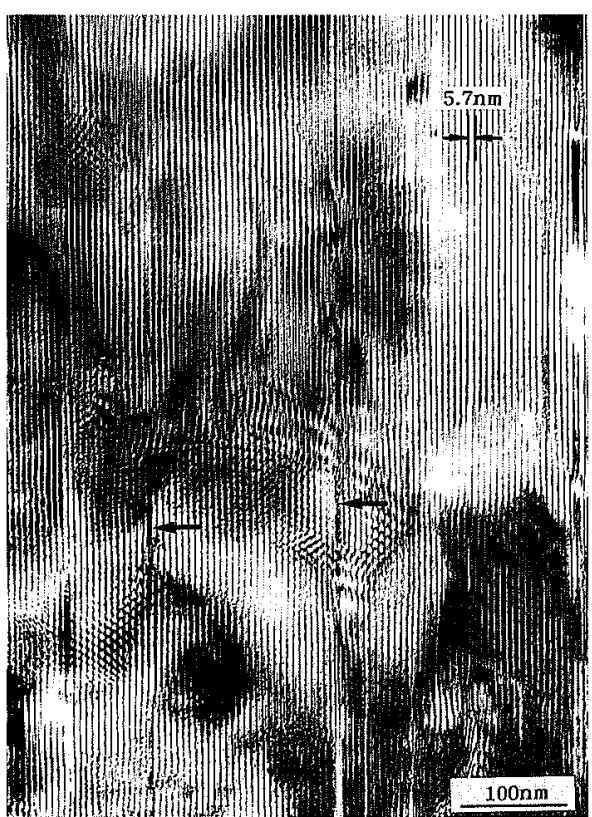

Fig. 9. Transmission electron micrograph showing (0001) lattice image. Stacking faults are shown by arrows.

images can be seen in addition to those from the ferrite. In this case, the $(0001)_{\delta}$ planes are inclined at about 3 and $11^{\circ}$ to the $(100)_{\alpha}$ plane. This indicates that the orientation relationship between the substrate ferrite and $\delta_{1}$-phase cannot be determined uniquely. It should, however, be pointed out that the $\delta_{1}$ crystals form always in contact with the substrate ferrite grain and the $\zeta$-phase crystals never exist between the $\delta_{1}$-phase and the ferrite. Figure 9 is the $(0001)_{\delta}$ lattice image of a $\delta_{1}$ crystal. Stacking faults parallel to the $(0001)_{\delta}$ plane are often observed as indicated by arrows.

\section{Discussions}

\subsection{Formation of $\zeta$-Phase Crystals}

Since the previous reports ${ }^{25}$ show that the $\zeta$-phase crystals nucleate at the interface between the initially formed $\mathrm{Al}$-enriched layer and the ferrite, the precipitation of them is thought to be a solid-phase reaction occurring via the diffusion of $\mathrm{Zn}$ atoms through the Al-enriched layer. In the present study, however, a thin layer comprising $\mathrm{Al}$ and $\mathrm{Fe}$ atoms between the $\eta$-phase and $\zeta$-phase layers could not be detected unfortunately. It should, however, be pointed out that the interface between the $\zeta$-phase crystals and the $\eta$-zinc film exhibits the sawteethlike morphology. At the very early stage of the Al-enriched layer formation, the surface of the substrate would be much more flat. Thus, this Alenriched layer was probably absorbed into the $\mathrm{Fe}-\mathrm{Zn}$ intermetallic $\zeta$ phase. It is also interesting to note that $\mathrm{Zn}$ concentration in the initially formed $\zeta$-phase crystals is the largest among those of $\mathrm{Fe}-\mathrm{Zn}$ intermetallics as has been reported previously. ${ }^{11)}$ In view of the Gibbs free energy-Composition diagram, Hillert ${ }^{26)}$ has pointed out that at the start of precipitation the phase which differs much in composition from the matrix may be favoured because of the larger driving force for the nucleation even if it cannot be in stable equilibrium with the matrix

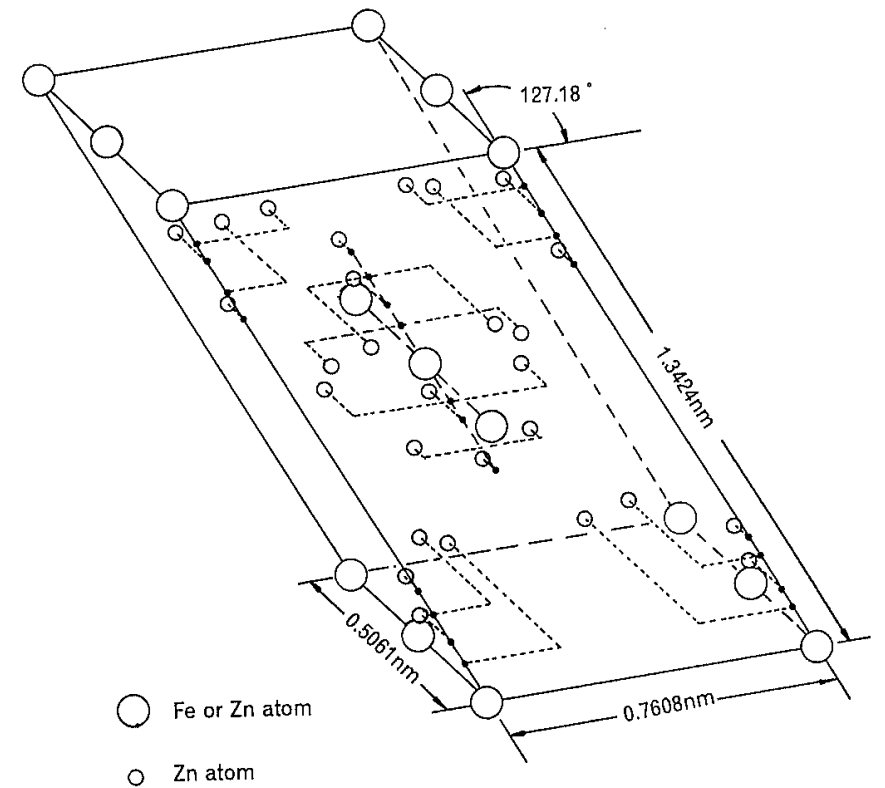

Fig. 10. Atomic configuration in a $\zeta$ lattice.

phase. This is in keeping with the present observation.

These intermetallics may grow epitaxially with ferrite as far as the atomic configuration of $\zeta$-phase lattice is close to that of ferrite. In fact, as has been demonstrated by Adachi et al., ${ }^{13)} \zeta$-phase crystals exhibit the rod-like morphology aligning in specific orientations with parallelogram cross sections also in the present study. The growth directions of these rods, however, are not parallel to $\langle 113\rangle_{\alpha}{ }^{13)}$ but parallel to $\langle 111\rangle_{\alpha}$ directions in the present study. The number of the growth directions of $\zeta$-phase crystals observed in the present study does not depend drastically on the substrate surface orientation as reported previously, ${ }^{13)}$ but varies with the location even within the same ferrite grain. If intermetallic crystals nucleate epitaxially at some places on a flat ferrite plane which is exactly parallel to a low Miller index plane such as $\{100\}_{\alpha},\{110\}_{\alpha},\{111\}_{\alpha}$ or $\{112\}_{\alpha}$, all the equivalent orientation relationships between the intermetallic phase and the substrate ferrite will be obtained. The restriction to a single growth direction is probably due to the slight misorientation of the substrate surface from a low index plane. Such a so-called "vicinal plane" probably consists of numerous mono-and/or several atomic height steps separated by terraces. If crystal growth occurs at such steps on a flat terrace with keeping atomic-site correspondence, the $\zeta$-crystals of a specific orientation will grow, leading to a single variant in the $\zeta / \alpha$ orientation relationship.

The orientation relationship between ferrite and $\zeta$-crystal could not be determined directly in the present study. It is, however, suggestive that the growth direction of $\zeta$-phase crystals was determined to be parallel to the $[001]_{\zeta}$ by Adachi et al ${ }^{13)}$ It should also be pointed out that the orientation relationship between $\zeta$-phase and ferrite obtained by them ${ }^{13)}$ is rather questionable because the thin foil prepared by means of diamond slicing was largely distorted. Thus in the present study a possible orientation relationship has been examined by comparing the atomic configurations of a $\zeta$-phase lattice 
and that of ferrite. The complex atomic arrangements within a unit cell of $\zeta$-phase lattice ${ }^{27)}$ is shown schematically in Fig. 10. Considering the growth direction of a $\zeta$-phase crystal is the $[\overline{1} 11]_{\alpha} / /[001]_{\zeta}$, the atomic configurations on a plane including this direction are examined. The good fit can be obtained in the case where $(101)_{\alpha} / /(100)_{\zeta}$ and $(12 \overline{1})_{\alpha} / /(010)_{\zeta}$. The atomic configuration on a $(101)_{\alpha}$ and that of the $(100)_{\zeta}$ plane on which the atoms of successive four atomic layers are projected are illustrated in Figs. 11(a) and 11(b), respectively. All

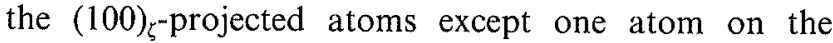
fourth layer in the unit cell of $\zeta$-phase lattice correspond to those on a $(101)_{\alpha}$. The atomic correspondence between both the crystals and the displacements of the atoms in

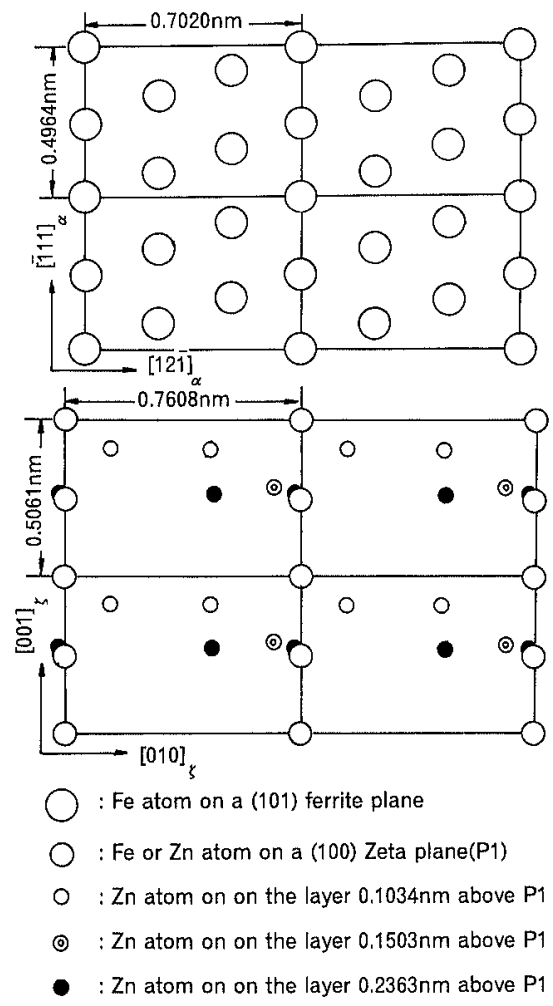

Fig. 11. Atomic configurations on the $(101)_{\alpha}$ and the $(100)_{5}$ planes.

(a) $(101)_{\alpha}$ and (b) the $(100)_{\zeta}$ plane on which the atomic sites of successive four atomic layers are projected.

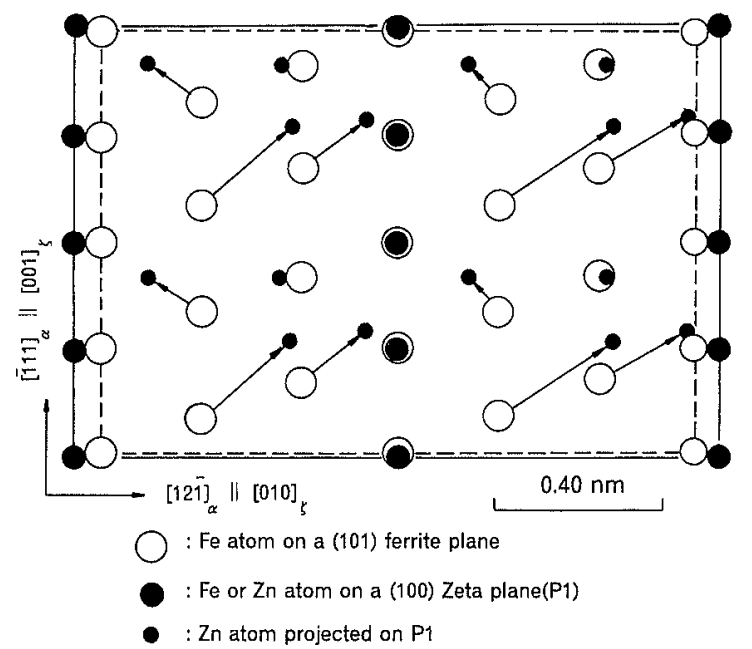

Fig. 12. Correspondence of the atomic configurations on the $(101)_{\alpha}$ and the $(100)_{\zeta}$ planes. the $(101)_{\alpha}$ plane necessary to construct the atomic configuration projected on the $(100)_{\zeta}$ is shown in Fig. 12. It can be seen that the misfit in the direction parallel to $[\overline{1} 11]_{\alpha} / /[001]_{\zeta}$ is as small as $1.9 \%$, implying that the growth direction of $\zeta$-phase crystals is probably parallel to this direction. The atomic configurations on the (101) is also quite similar to the $(100)_{\zeta}$ plane. Thus the parallelism between these two planes will lead to the following orientation relationship.

$$
\begin{gathered}
{[\overline{1} 11]_{\alpha} / /[001]_{\zeta}} \\
(101)_{\alpha} / /(100)_{\zeta} \\
(12 \overline{1})_{\alpha} / /(010)_{\zeta}
\end{gathered}
$$

\subsection{The Formation of $\delta_{1}$-Phase Crystals}

On the cross sections of as-hot dip galvanized sheets, only $\zeta$-phase layer was detected by both scanning electron microscopy and $\mathrm{X}$-ray diffraction as an intermediate intermetallic layer. In the cross-sectional TEM observation, however, $\delta_{1}$-phase crystals were recognized in contact with ferrite. The formation of $\delta_{1}$-phase crystals in this case is probably due to the additional heat cycle during thin foil preparation. That is, the specimen consisting of stacked several hot-dipped sheets was again immersed into the $\mathrm{Zn}$-bath kept at $723 \mathrm{~K}$ for $2 \mathrm{~s}$ to bond the interfaces by remelting $\mathrm{Zn}$ surfaces. Since $\mathrm{Zn}$ content in $\delta_{1}$-phase is much lower than that in $\zeta$-phase, i.e., $\mathrm{FeZn}_{7}$, it is likely that $\delta_{1}$-phase crystals form at the interface between $\zeta$-phase layer and ferrite as the result of thermodynamic consideration ${ }^{26)}$ described above. It should also be noted that further isothermal holding at $723 \mathrm{~K}$ or higher temperatures may result in the formation of $\Gamma$ and/or $\Gamma_{1}$ phases. In the present study without an additional heat treatment such as galvannealing, however, only two intermetallic phases, $\zeta$ and $\delta_{1}$ were detected in the as-hot dip galvanized and in the TEM specimens, respectively.

The $\delta_{1} / \alpha$ or the $\delta_{1} / \zeta$ orientation relationship cannot be determined uniquely and the $\delta_{1}$-phase crystals in different orientations were observed in contact with a ferrite grain as in Figs. 7,8 and 9. This implies that these crystals did not grow epitaxially with either a ferrite or a $\zeta$-phase crystal. Such a non-epitaxial nucleation of $\delta_{1}$-phase crystals may be due to significantly large differences in atomic configurations among these lattices.

\subsection{Out-burst Reaction}

As shown in Fig. 3, some parts of the $\zeta$-phase layer broke into the fragments which disperse into the $\eta$-zinc layer were also often observed. Such a phenomena is referred to as the out-burst reaction. ${ }^{23,24)}$ Considering its morphology, it is likely that this reaction occurs during the stage where the $\eta$-zinc layer is still in viscous state. If the $\zeta$-phase crystals nucleated at the steps on the substrate ferrite surface and grew into the viscous $\eta$-zinc layer with absorbing $\mathrm{Fe}$ atoms dissolved in the molten $\mathrm{Zn}$, the out-burst reaction could not occur because any constraint inducing the elastic stress to break the $\zeta$-phase crystals does not exist. Thus, the growth of $\zeta$-phase crystals should occur as a solid-phase reaction within the ferrite via the diffusion of $\mathrm{Zn}$ atoms into the ferrite lattice as explained in the preceding section, i.e., $\mathrm{Zn}$ atoms 
penetrate into the ferrite lattice as substitutional atoms and form the $\zeta$-phase lattice. If these $\mathrm{Zn}$-enriched ferrite regions are transformed to the $\zeta$-phase lattices with keeping the interphase boundary coherency, the volume expansion of about $25 \%$ due to this $\zeta$-formation will result in a quite large elastic strain. Furthermore, since an intermetallic phase is normally very brittle, such a large compressive stress will lead to the fracture of the $\zeta$-phase layer, resulting in the out-burst reaction. ${ }^{23,24)}$ It should also be noted that the $\zeta / \alpha$ orientation relationship produces anisotropic elastic stress. The misfits along the $[001]_{\zeta} / /[\overline{1} 11]_{\alpha}$, the $[010]_{\zeta} / /[12 \overline{1}]_{\alpha}$ directions and the direction normal to the $(100)_{\zeta} / /(101)_{\alpha}$ plane are 1.95 , 8.38 and $12.93 \%$, respectively. Therefore, if the ferrite substrate surface involves the direction normal to the $(100)_{\zeta} / /(101)_{\alpha}$ plane and parallel to the $[010]_{\zeta} / /[12 \overline{1}]_{\alpha}$, i.e., the substrate surface is parallel to the $(\overline{1} 11)_{\alpha}$, the compressive stress acting on the $\zeta$-phase layer will be the maximum and the out-burst reaction will occur quite frequently. Thus, it can be expected that the occurrence of the out-burst is reduced by choosing the ferrite substrate surface close to $\{110\}_{\alpha}$, the largest expansion due to the $\zeta$-phase formation being in the direction normal to the surface. It should be mentioned here that, although the occurrence of the out-burst reaction has been explained in terms $\zeta$-phase precipitation, the occurrence of this reaction is also possible via the formation of other $\mathrm{Fe}-\mathrm{Zn}$ intermetallics as well.

\section{Conclusions}

The formation of intermetallic interphase boundary layers in a hot-dip galvanizing steel sheet has been examined and the following results have been obtained.

(1) The initially formed interphase layer is $\zeta$-phase crystals which form via a solid phase reaction with the diffusion of $\mathrm{Zn}$ atoms into the substrate ferrite.

(2) Considering the atomic configurations of the $\zeta$-phase crystal, these $\zeta$-phase crystals are thought to be related to the ferrite with the following orientation relationship:

$$
\begin{gathered}
{[001]_{\zeta} / /[\overline{1} 11]_{\alpha}} \\
(100)_{\zeta} / /(101)_{\alpha} \\
(010)_{\zeta} / /(12 \overline{1})_{\alpha}
\end{gathered}
$$

The growth direction of a $\zeta$-phase crystal is probably parallel to $[001]_{\zeta} / /[\overline{1} 11]_{x}$.

(3) Additional short period immersion in the $\mathrm{Zn}$ bath produces a $\delta_{1}$-phase layer between the $\zeta$-phase layer and the ferrite. Any specific orientation relationship cannot be observed between $\delta_{1}$-phase and either $\zeta$-phase or ferrite.

(4) The lattice image of $(0001)_{\zeta}$ planes can easily be observed with the stacking faults parallel to this plane.

(5) The out-burst reaction is thought to arise from the brittle fracture of some parts of $\zeta$-phase layer and the dispersion of the fragments into viscous $\mathrm{Zn}$ due to the large compressive stress within this layer arising from the coherency between $\zeta$-phase and the ferrite.

\section{Acknowledgements}

This research was carried out as a part of the research programme of the Basic Research Committee of Interphase Compounds in the Surface-treated Steels of The Iron and Steel Institute of Japan. The authors would like to express their sincere thanks to The Iron and Steel Institute of Japan for the financial support for this research. Thanks are also due to the members of the Advanced Instrumentation Center, Ehime University for providing a JEOL $2000 \mathrm{EX}$ electron microscope.

\section{REFERENCES}

1) J. M. Keen and J. P. G. Farr: J. Electrochem. Soc., 109 (1962), 668.

2) J. O' M. Bockris and H. R. Thirsk: Fundamental Aspects of Electrocrystallization, Prenum Press, New York, (1967).

3) J. O' M. Bockris: J. Electrochem. Soc., 116 (1969), 1503.

4) B. S. Sheshadri and T. H. V. Setty: Electrochim. Acta, 17 (1972), 1895 .

5) T. Seiyama, S. Itoh, N. Yamazoe and G. Okada: Denki Kagaku, 40 (1972), 748.

6) S. Itoh, G. Okada and T. Seiyama: Denki Kagaku, 42 (1974), 242.

7) K. Kamei and Y. Ohmori: J. Appl. Electrochem., 17 (1987), 821.

8) Y. Ohmori, K. Kondo, K. Kamei and S. Hinotani: Mater. Res. Soc. Symp. Proc., Vol. I22, Materials Research Society, (1988), 553.

9) Y. Ohmori, K. Nakai, H. Ohtsubo, T. Yagi and T. Matsumoto: ISIJ Int., 33 (1993), I 196.

10) H. Ohtsubo, T. Matsumoto, K. Nakai and Y. Ohmori: ISIJ Int., 34 (1994), 1002

I1) Y. Wakamatsu and M. Onishi: Tetsu-to-Hagané, 64 (1978), 2215.

12) E. Scheil: Z. Metallkd., 27 (1935), 76.

13) Y. Adachi, T. Nakamori and K. Kamei: J. Jpn. Inst. Met., 56 (1992), 1235

14) Y. Adachi, M. Arai and T. Nakamori: Tetsu-to-Hagané, 80 (I994), 225

15) Y. Adachi, M. Arai and T. Nakamori: Tetsu-to-Hagané, 80 (1994), 647.

16) D. Horstmann: Arch. Eisenhüttenwes., 27 (1956), 297.

17) L. A. Giannuzzi, A. S. Ramani, P. R. Howell, H. W. Pickering and W. R. Bitler: Zinc-Based Steel Coating Systems: Metallurgy and Performance, ed. by G. Krauss and D. Matlock, TMS, (1990), 121 .

18) L. A. Giannuzzi, A. S. Ramani, P. R. Howell, H. W. Pickering and W. R. Bitler: Proc. 50th Annual Meeting of Electron Microscopy of America, ed. by G. W. Bailey and J. A. Small, San Francisco Press, (1992), 36.

19) L. A. Giannuzzi, P. R. Howell, H. W. Pickering and W. R. Bitler: Proc. Mater. Res. Soc. Symp., 254 (1992), 159.

20) L. A. Giannuzzi, A. S. Ramani, P. R. Howell, H. W. Pickering and W. R. Bitler: Mater. Charact., 30 (1993), 54.

21) Y. Lin, Wen-An Chiou and M. Meshii: Proc. 49th Annual Meeting of Electron Microscopy of America, ed. by G. W. Bailey, San Francisco Press, (1991), 568.

22) M. Onishi, Y. Wakamatsu and H. Miura: Trans. JIM, 15 (1974), 331 .

23) A. Nishimoto, J. Inagaki and K. Nakaoka: Tetsu-to-Hagané, 68 (1982), 1404.

24) A. Nishimoto, J. Inagaki and K. Nakaoka: Tetsu-to-Hagané, 72 (1986), 989

25) M. Saito, Y. Uchida, T. Kittaka, Y. Hirose and Y. Hisamitsu: Tetsu-to-Hagané, 77 (1991), 947.

26) M. Hillert: Lectures on the Theory of Phase Transformations, ed. by H. I. Aaronson, AIME, New York, (1977), 1.

27) W. B. Pearson: A Handbook of LATTICE SPACINGS AND STRUCTURES OF METALS AND ALLOYS, Pergamon Press, New York, (1967), 255. 